\title{
AN ALOGRITHM FOR DIAGNOSIS OF AUTISM BASED ON FACIAL IMAGES
}

\author{
Sharwan K Dewan \\ 1167, Sector-1, Rohtak, PIN-124001, India \\ E mail ID: Sharwandewan@rediffmail.com
}

\begin{abstract}
Diagnosis of autism is very intricate and is based on the answers given by parents or guardians to questionnaire. In view of the difficulties encountered in the diagnosis, the idea of an algorithm based on facial images for the diagnosis of autism is advanced to make the diagnosis very simple, easier, accurate and rapid.

KEY WORDS: Autism, Alogrithm , Algorithm For Autism, Facial Technology, DNN, VGGFace, Autistic, Nonautistic
\end{abstract}

\section{INTRODUCTION}

Autism is a brain developmental disorder that affects about one in 68 children. The child with autism lacks social communication, eye contact, and has difficulties with cognition and perception. Diagnosis of autistic children goes unnoticed by the parents and even by the untrained doctors throughout the world. Early intervention is known to provide the necessary relief to such children and their parents. Thus, that is always a welcome step if the diagnosis of such children could be made easy, simplified and time saving as well.

In the view of this author, artificial intelligence can possibly be used to identify autistic people from among autistic and nonautistic based simply on their facial images alone.

Facial recognition technology, an artificial intelligence technique has been known for quite sometime now. In fact, that technology is becoming not only enormously speedy and reliable but also accurate. That can be guessed from its usage as a security feature in the latest smartphones the world over. That technology is also being used by different governments in the world to tackle crime in their countries.

Evidently, it can be concluded that human faces seem to contain much more useful discriminative information that could be extracted out easily through the alogrithmic technology than otherwise.

A large number of pictures of self-identified autistic and nonautistic people can be fed into an algorithm that could be trained to learn the subtle differences in their features. In recent years deep neural networks, DNNs are being widely used in artificial intelligence. DNNs mimic the brains neurocortex as they are able to stimulate large, multilevel networks of interconnected neurons. In fact, they outperform humans in visual tasks like large scale analysis, classification, facial recognition, speech recognition, visual object detection, pattern extraction, and even in diagnosing skin cancer etc.

Neural networks are a set of algorithms, modeled loosely after the human brain. They are designed to recognize patterns in any given data. These networks interpret sensory data through a kind of machine perception, labeling or clustering raw input. They help to group unlabeled data 
according to similarities among the example inputs. They classify the given data when they have a labeled dataset to train on. They help us cluster and classify. They serve as a clustering and classification layer on top of data we store and manage. In other words, DNNs extract features that are fed to other algorithms for clustering as well as classification. We can regard them as components of larger machine-learning applications involving algorithms for reinforcing learning, classification, as well as regression.

Certain trends can be identified from among the data regarding the differences in the facial features between the two categories of children-autistic and nonautistic for bith the genders-boys and girls separately. For example, eyes,eye lashes. mouth, mouth corners, chin, nose, forheads, jaws etc. We have identified some differences in certain characteristics between the two categories but the data we have are too small to confirm. And that needs to be confirmed by the DNNs on a very large number of the facial images data.

Facial features can be extracted using a DNN that can be specifically developed such that it concentrates upon non- transient features of the facial images while excluding the heads orientation as well as the background in the images. The location of the face in the image, outlines of its elements, and the head's orientation can be extracted using a commomnly used face-detection software: Face++. One of the most common softwares, Face ++ can be employed that focusses on the areas of the faces while ignoring the background. In this software, facial landmarks, pitch, roll and yaw parameters are used to describe the orientation of heads in space. The facial landmarks point out the location of the facial landmarks outlining the contour and elements of the face. In fact, DNNs can recognize patterns in large unstructured data, for instance, in digital images, sound or text. Not only that such patterns can be analysed as well to make ptedictions. While human brain is subject to either missing or misunderstanding the links between characteristics and facial features, the DNNs do not, thus displaying their all important role in artificial intelligence and computer vision. The age factor would have to be balanced. To start with, the DNN algorithm would have to be trained for the two years and then higher age groups. Later the work could be extende to one year and six months age and finally to as low as one day age facial images. This scheme applies separately to each gender, boys and girls.

Facial features can be extracted from the images using a widely employed DNN, called VGGFace . It should be noted that the DNN, VGG-Face was originally trained and developed employing a sample of 2.6 million 246 images for the purpose of facial recognition. In other words,for recognizing a given person across different 247 images). Note that the VGG-Face is similar to traditional scoring keys accompanying psychometric tests.

The prediction model, logistic regression, combined with a standard dimensionality-reduction approach can be used for training classifiers, the singular value decomposition (SVD). The models would have to be trained separately for each gender, boys and girls. The autism diagnosis done using the questionnaire could be used as a dependent variable. The developed DNN can then be used for predicting rather diagnosing autism from facial images alone in children aged two years or higher and finally to infants at six months and then to babies aged one month etc and finally to babies right at birth.

To start with, the work can be initiated in the western world and then extended to the nonwestern world as images of a large number of children, autistic (and nonautistic) of different age groups would be required which could be perhaps readily available in the western world. 
DOI : https://dx.doi.org/10.26808/rs.ph.i7v5.04

International Journal of Pharmaceutical Science and Health

Issue 7, Vol. 5 (September- October 2017)

Available online on http://www.rspublication.com/ijphc/index.html

ISSN $2249-5738$

\section{CONCLUSIONS}

To conclude, the usage of deep neural networks for diagnosis of autism from facial images alone has been suggested as a simple, easy, effective, and accurate tool. That might prove its worth for the diagnosis of a baby with autism right at birth.

\section{REFERENCES}

Parkhi, O. M., Vedaldi, A., \& Zisserman, A. (2015). Deep Face Recognition. In X. Xie, M. W.Jones, \& G. K. L. Tam (Eds.), Proceedings of the British Machine Vision Conference (p.795 41.1). Swansea, UK: BMVA Press. http://doi.org/10.5244/C.29.41 